\title{
Globally Control Robot for Surveillance
}

\author{
M. Lokeshbabu*, T. S. Balaji, B. Kamalesh and P. Pramod \\ Department of Electronics and Communication, SRM University, 100 Feet Road, Vadapalani, \\ Chennai -600026, Tamil Nadu, India; lokeshmadala16@gmail.com, balaji1381@gmail.com, \\ sachinkamalesh@gmail.com, pramodpullela@gmail.com
}

\section{Abstract}

Objective: This paper mainly deals with controlling the robot using internet and camera is used for monitoring purpose. Methods/Analysis: Surveillance robots can be operated either by PC or mobile. The main purpose of the paper is to monitor surroundings by controlling a robot by user over a wide range which can be obtained by using mobile connecting to the internet. Findings: This paper uses raspberry pi 2 to make a robot which minimizes the wiring. Raspberry pi 2 implemented robots can be operated with in a hotspot range using mobile without internet facility. To increase the range, it is necessary to connect the mobile with internet. When it is connected to the internet, the robots can be controlled by human operator over a large distance. Novelty/Improvements: Wide range and large distance coverage can be obtained by using this method.

Keywords: Internet, Surveillance Camera, System on Chip

\section{Introduction}

As robots and their peripheral equipment become more sophisticated, reliable and miniaturized, these systems are increasingly being utilized for military and law enforcement purposes.

Internet of things is changing much about the world we live in. Sophisticated sensor and chips are embedded in physical element that surround us each transmitting a valuable data that could be understand how it works and work together". But how exactly do all these devices share such large quantities of data how to implement their works whether improving production of factory, giving city residents real time updates where to park on, monitoring our personnel health if the common IoT platform that brings this devices information together and provide common language for the devices and apt to communicate with each other. The process starts with themselves which securely communicate with an IoT platform. This platform integrates the data from many devices and applies analytics to share the most valuable data with applications.

Considering the situation that, a robot controls through IoT. A component in raspberry pi called bus enumerator which gathers data from all devices and sensors then passes it to a gateway in PC. Through gateway most relevant information is transmitted, before sending this organized data to the PC gateway, both must be registered with each other and confirm a secure communication.

There are two different ways to control a robot i.e. by wired and wireless. There are lots of technologies used for controlling a robot based on distance i.e. short range and larger range. For short range most efficient way to control a robot in a secured way is by NFC technology. It can operate a robot using DTMF technology . As it has some disadvantages further ZIGBEE, Bluetooth technologies are developed and they are useful for shorter range and they produce a delay, in order to overcome these limitations Wi-Fi technology was introduced. Wi-Fi technology can be used in range of RF as well as it can be widely used through internet. As we are using Wi-Fi technology over a wide range so that robot can be controlled through internet by assigning an IP address. In order to assign an IP address most efficient way is to use a raspberry pi whose cost is less than 40 dollars. Wired connection can be minimized as raspberry pi is preprogrammed SOC. 


\section{Existing System}

Radio frequency technology is a wireless communication system that can be used to transmit different types of signals from transmitting end to receiving end. In the existing system, the radio frequency signals or radio waves are carried out to take up for processing a certain application.

\subsection{Radio Frequency Remote Control Robot} A robotic vehicle is designed and it is properly interfaced and controlled by radio frequency remote control, so that this kind of vehicle is named it as radio frequency controlled robotic vehicle. A control panel is facilitated with RF transmitter and receiver which will be interfaced to the robotic vehicle and whatever the controlling instructions passing from the RF transmitter is delivered and executed with the help of RF receiver. By selecting a proper antenna, the robotic vehicle can be controlled with the help of radio frequency remote control ${ }^{\text {. The entire }}$ process of controlling the robotic vehicle can be carried out with this radio frequency remote control technology. For destroying or diffusing a distant object, a 8051 microcontroller is interfaced with low-power laser light and this done for demonstration purpose. For the better understand of this paper it is necessary to learn about RF modules.

\subsection{Transmitter}

A compact size model of RF modules is used to transmit the logic 0 and logic 1 depends upon the signal which will be required to transmit to the robotic vehicle. The robotic vehicle which is acts as a receiver and it is properly interfaced with 8051 microcontrollers and the transmitter. $3 \mathrm{~V}$ to $6 \mathrm{~V}$ is the supply voltage range of the $\mathrm{RF}$ transmitter and $4 \mathrm{~V}$ to $12 \mathrm{~V}$ with a frequency of 433.92 $\mathrm{MHz}$ is the output power range.

Figure 1 robotic vehicle receives the signal from the RF transmitter which will facilitated with push buttons that is for the purpose of movement in various specified direction described by the user. The controlling action of robotic vehicle is decided by the push buttons present in the RF transmitter ${ }^{3}$.

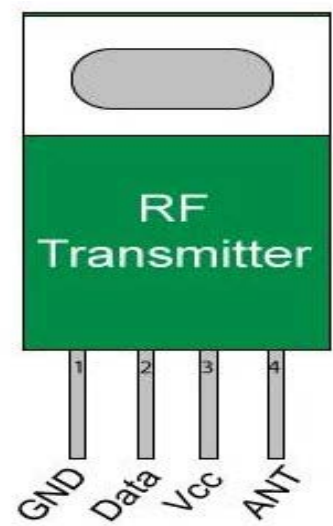

Figure 1. RF transmitter.

\subsection{Receiver}

$\mathrm{RF}$ receiver is also designed to work in a same frequency like RF transmitter so that the co-ordination among these two will be happened successfully which will lead to produce perfect communication between the transmitter and receiver.

By pressing the push buttons the data is collected by RF transmitter is ten encoded and transmitted to the distance where the robotic vehicle is located. The robotic vehicle is located at the desired distance with a proper RF antenna and it is ready to decode the data which will be sending by RF transmitterf. Once the data is decoded it sends the control to the following two things; one is driving the DC motors with the help of microcontroller and another one is motor driver IC to move the robotic vehicle.

Once the RF transmitter transmits the signal that will be received by the robotic vehicle which is interfaced with microcontroller mounted on a RF transmitter. DTMF technology is one among the way of controlling the robotic vehicle. Many techniques were involved for controlling the robotic vehicle either for longer range or shorter range. For longer range, the robotic vehicle is controlled through mobile phone?

\section{Proposed System}

Robot is controlling using IOT concept that is through internet. Because of the available technique, it is possible to operate the robot universally (Figure 2 and 3 ). In the 
design using $\mathrm{dc}$ motors which cannot be connected directly to raspberry pi GPIO pins. As the output voltage at raspberry pi is less so that the motors cannot be driven, in order to drive the motor, it is mandatory that motor driver IC L293D which can interface with raspberry pi. As it is a preprogrammed module with lot of options. It contains ARM cortex a7 processor where in existed system we used 8051 microcontroller shown in Table 1. The major requirement in this proposed system is that, the controlling action should e done immediately whenever receives the signal, communication between the transmitter and receiver must be efficient and many other functions like ADC, PWM are required along with large memory requirement. It is found that ARM processor is the best fit for the above specified requirement.

\section{BLOCK DIAGRAM}

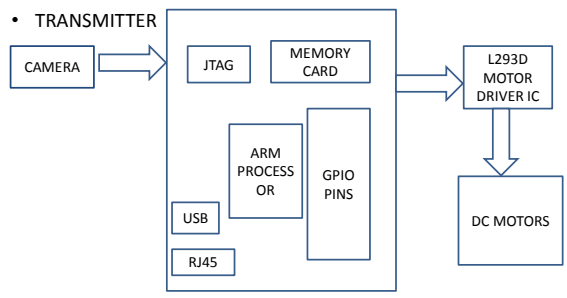

Figure 2. Transmitter block.

RECEIVER

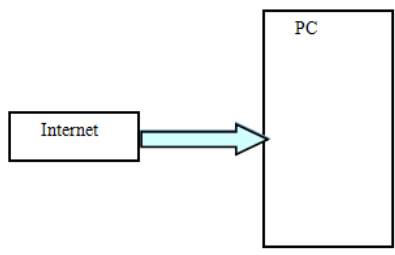

Figure 3. Receiver block.
It is possible to connect internet to raspberry pi by assigning an IP address directly. Here to control robot by using putty software which provide serial secure connection? A camera is attached to raspberry pi at CSI module. By using this, it can monitor surrounding which can viewed in PC using a browser by typing raspberry pi IP address.

\section{Experimental Result}

The experimental output is shown in Figure 4, which explains about how the raspberry pi assigned with an IP address through which monitoring and controlling of the robot action takes place.

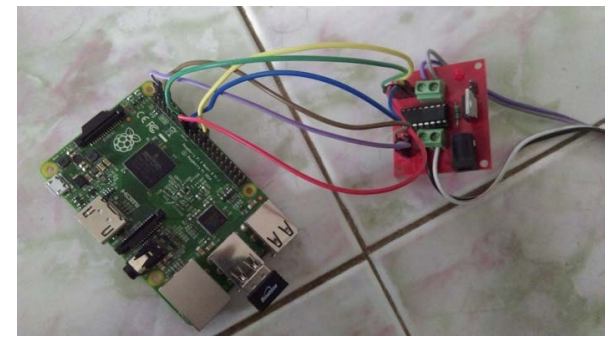

Figure 4. Experimental output.

\section{Algorithm}

Step1: Initiaizing the library files.

Step2: Setting the value for left motor, right motor speed and acceleration to zero and assigning maximum and minimum speed values.

Step3: Interfacing raspberry pi with L293D IC by assigning GPIO pins and PWM setting.

Step4: Assign 1001 for forward movement, 0110 for reverse movement \& 0000 to stop.

Table 1. Comparison between arm and 8051 microcontroller

\begin{tabular}{lll}
\hline Parameters & ARM & Microcontroller \\
\hline Bus width & Available in 32 and 64 bit & Available in 8 bit \\
Communication protocols & UART,USART,I2C,SPI,CAN,USB,ETHERNET,DSP,SAI & UART,USART,I2C,SPI \\
Speed & 1 clock/instruction cycle & 12clock/instruction cycle \\
Memory & Flash,SDRAM,EEPROM & SRAM,ROM,FLASH \\
ISA & RISC & CISC \\
Memory Architechture & Modified Harvard architecture & Modified Neumann architecture \\
Power consumption & Low & Average \\
Families & ARM-4,5,6,CORTEX SERIES & 8051 VARIANTS \\
COMMUNITY & VAST & VAST \\
COST & LOW & VERY LOW \\
Other feature & High Speed operations & For standard operations \\
Manufacturers & Apple,Qualcomm & Atmel,silicon labs \\
\hline
\end{tabular}


Step5: Find the value of acceleration then it will take following decisions accordingly

Acceleration $>0$; minimum to maximum speed $=$ acceleration.

Acceleration $<0$; minimum speed=acceleration.

Acceleration $>$ maximum speed; maximum speed $=$ acceleration.

Step6: Comparing motor speeds to control the movement of robot to left or right.

Step7: For the controlling actions the assigned values are such that $\mathrm{W}$ for forward, $\mathrm{S}$ for reverse, $\mathrm{A}$ for left, $\mathrm{D}$ for right, Q for stop and ctrl+ $\mathrm{x}$ to stop completely, which are commands programmed in putty software for movement of robot.

\section{Conclusion}

The designed robot can be universally controlled through internet by assigning IP address to raspberry pi 2, using that IP, possible to view the live streaming in browser of the surrounding what exactly to be monitored. In order to connect to particular network, it can be programmed it by writing a username and password of particular network in to it. Using of raspberry pi 2 that reducing the complexity. It can be used for military surveillance and it can also operate by connecting to a local network without using internet. Power consumption is less when compared to existing system.

\section{References}

1. Kinnal RV, kudari S, Kachavimath AV. Control of vehicle pollution through internet of things and traffic lights. International Journal of Emerging Technology in Computer Science and Electronics. 2015 Apr; 14(2):227-31.

2. Cho YC, Jeon JW. Remote robot control system based on DTMF of mobile phone. The IEEE International Conference on Industrial Informatics (INDIN 2008) DCC, 2008 Jul 13-16, Daejeon, Korea; 2008. p. 1441-6.

3. Singh AK, Shanker NM, Yadav AP. RF controlled terrorist fighting robot. International Journal of Computer Science and Communication. 2010 Jan-Jun; 1(1):109-12.

4. Divya V, Dharanya S, Shaheen S, Umamakeswari A. Amphibious surveillance robot with smart sensor nodes. Indian Journal of Science and Technology. 2013 May; 6(5):4496-9.

5. Masehian E, Katebi Y. Robot motion planning in dynamic environments with moving obstacles and target. International Journal of Mechanical Systems Engineering. 2007; $1(1): 20-5$. 